\title{
Crossover-behavior of the saturation magnetic fields of (100) and (110) $\mathrm{Fe} / \mathrm{Cr} / \mathrm{Fe}$ trilayers
}

\author{
C. Chesman, C.G. Bezerra*, E.L. Albuquerque, A.M. Mariz \\ Departamento de Física, Universidade Federal do Rio Grande do Norte, 59072-970 Natal, RN, Brazil \\ Received 9 June 2005; received in revised form 18 January 2006; accepted 21 January 2006 \\ Available online 27 January 2006 \\ Communicated by R. Wu
}

\begin{abstract}
We use a simple and realistic theoretical model to investigate the saturation magnetic fields of ultrathin (100) and (110) Fe/Cr/Fe trilayer system. We consider the Fe films presenting magneto-crystalline anisotropies (cubic and uniaxial) and interacting through bilinear and biquadratic exchange couplings. From the equilibrium configurations, which are determined by the absolute minimum of the magnetic energy, we were able to set up field-dependent phase diagrams for the situation where the external static magnetic field $H$ is applied in the plane of the films at an arbitrary angle $\theta_{H}$. We have obtained the critical frontier between the saturated and non-saturated phases. Two main results should be pointed out: (i) the change of the symmetry axis according to the magneto-crystalline fields and (ii) a sudden change (on a multicritical point) of the character of the magnetic phase transitions, typical of a crossover behavior.

(C) 2006 Elsevier B.V. All rights reserved.
\end{abstract}

PACS: 75.70.Cn; 75.30.Gw; 71.70.Gm; 75.75.+a

In the last decade a number of papers have been focused on the understanding of magnetic properties of layered films of ferromagnetic metals exchange coupled through non-magnetic spacer layers (see Ref. [1] for a review). In particular, a very simple and interesting situation occurs when only two ferromagnetic films are coupled through one non-magnetic spacer, the so-called trilayer system. Despite its simplicity, such a system presents a very rich physical behavior, and numerous attempts were taken by many authors to plot the phase diagram of biquadratically coupled trilayer structures so far (see, for instance, Refs. [2-4]). Besides, these devices are excellent options for technological applications and attractive objects of research.

The determination of the equilibrium configuration, as a function of the various terms which compose the total free magnetic energy of the system, is very significant to understand how an external input can affect the trilayer structure. For example,

\footnotetext{
* Corresponding author.

E-mail address: cbezerra@dfte.ufrn.br (C.G. Bezerra).
}

recently it was observed the creation of magnetic bridges in epitaxial $\mathrm{Fe} / \mathrm{Cr} / \mathrm{Fe}(001)$ trilayers caused by the bombardment of a $5 \mathrm{keV}$ He ion beam, producing an enhancement of the antiferromagnetic interlayer coupling strength, as the thickness of the $\mathrm{Cr}$ spacers is increased [5]. However, difficulties can arise if one is dealing with actual theoretical calculations, being easier to introduce the concept of magnetic configurations, which in turn is of crucial importance to define, among other magnetic physical parameters, the interlayer exchange energy (IEC) and the (giant) magnetoresistance (GMR) [6]. As a consequence, their corresponding phase diagrams play an important role from both the theoretical and experimental points of view.

In recent years many efforts have been done in constructing field-dependent phase diagrams of magnetic multilayers and superlattices. For example, Almeida and Mills [7] have studied the influence of the biquadratic exchange coupling on the features of the phase diagrams of infinite and finite superlattices. They reported the importance of both the magneto-crystalline anisotropy (uniaxial type) and biquadratic exchange coupling on the nature of the phase transitions, induced by the external applied magnetic field and magnetic configurations. Further- 


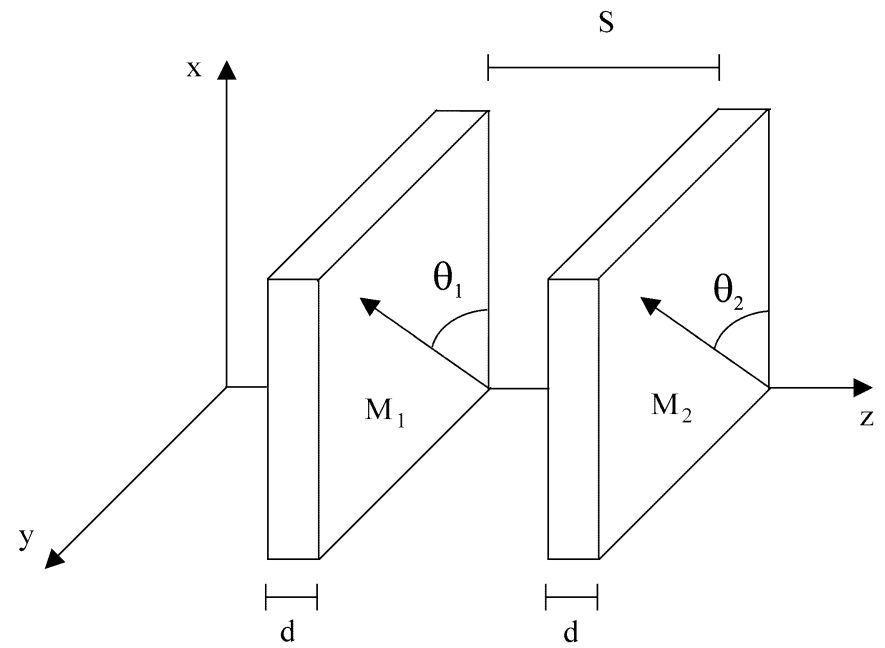

Fig. 1. Schematic representation of the trilayer structure and coordinate system considered in this Letter.

more, they also have found a very interesting even-odd effect (considering the number of magnetic layers) related to a spinflop phase. Later, phase diagrams of magnetic superlattices presenting cubic anisotropy instead of a uniaxial one, showed a totally different symmetry and, consequently, a quite different behavior [8]. Finally, Chesman et al. [9] presented very interesting phase diagrams of trilayer systems with cubic anisotropy and biquadratic exchange coupling. From their results it is possible to find criteria for measurements of the bilinear and biquadratic exchange couplings.

It is well known that, in general, it is difficult to obtain analytical expressions for the transition and saturation magnetic fields. This is because the free magnetic energy may present several terms, each one with a different influence on the transition and saturation magnetic fields. However, numerical results are much easier to obtain and furnish reliable and useful information for experimentalists. In particular, from a practical point of view for technological applications, the saturation magnetic field is an essential information.

The motivation of this work is a contribution to the determination of the saturation magnetic fields of trilayer structures when the system presents both uniaxial and cubic anisotropies. We have studied $\mathrm{Fe} / \mathrm{Cr} / \mathrm{Fe}$ trilayers in two possible different crystallographic orientations, namely (100) and (110). We should remark that the most part of the research performed about trilayer systems has been focused on (100) structures. Therefore, there is a lack of studies considering trilayer systems grown in different crystallographic orientations, for example, the (110) direction.

The geometry and the coordinate system employed here are shown in Fig. 1. We consider two magnetic single-crystal films, 1 and 2, having cubic lattice structure and thickness $d$ separated by a non-magnetic spacer layer with thickness $S$. The very strong demagnetization field, generates by tipping the magnetization out of plane, will suppress any tendency for the magnetization to tilt out of plane. The global behavior of the system is well described by a simple theory in terms of the mag- netic energy per unit area [10], i.e.,

$E_{T}=E_{Z}+E_{c a}+E_{u a}+E_{b l}+E_{b q}$.

Here $E_{Z}$ is the Zeeman energy (between the ferromagnetic films and the external applied field), $E_{c a}$ (cubic anisotropy) and $E_{u a}$ (uniaxial anisotropy) are the magneto-crystalline anisotropy energies, which we assume present in the ferromagnetic films. Also, $E_{b l}$ and $E_{b q}$ are the bilinear and biquadratic exchange energies (coupling the ferromagnetic films). It is usual to write the total magnetic energy per unit area in terms of experimental parameters (or effective fields) of each component of the magnetic energy [9,10]. For example, considering the (100) growth direction we get

$$
\begin{aligned}
\frac{E_{T}}{d M_{S}}= & -\sum_{i=1}^{2} H \cos \left(\theta_{i}-\theta_{H}\right) \\
& +\sum_{i=1}^{2}\left[\frac{1}{8} H_{c a} \sin ^{2}\left(2 \theta_{i}\right)-\frac{1}{2} H_{u a} \cos ^{2}\left(\theta_{i}-\theta_{u}\right)\right] \\
& -H_{b l} \cos \left(\theta_{1}-\theta_{2}\right)+H_{b q} \cos ^{2}\left(\theta_{1}-\theta_{2}\right) .
\end{aligned}
$$

Here, $H$ is the external magnetic field which is applied in the film plane, $M_{S}$ is the saturation magnetization and $H_{c a}\left(H_{u a}\right)$ is the cubic (uniaxial) anisotropy field, respectively. Also, $H_{b l}$ is the bilinear exchange coupling field which favors antiferromagnetic alignment (ferromagnetic alignment) if negative (positive), $H_{b q}$ is the biquadratic exchange field, which is responsible for a $90^{\circ}$ alignment between two adjacent magnetizations and is experimentally found to be positive, and $\theta_{H}\left(\theta_{u}\right)$ is the external magnetic field (uniaxial anisotropy) orientation. For the case of (110) growth direction, the total magnetic energy per unit area is expressed in Eq. (2), provided the term of the cubic anisotropy is replaced by [11]

$\sum_{i=1}^{2} \frac{1}{8} H_{c a}\left[\sin ^{2}\left(2 \theta_{i}\right)+\cos ^{4}\left(\theta_{i}\right)\right]$.

Once the free magnetic energy is determined, we can calculate the equilibrium configurations for specific values of the experimental parameters and external applied field. In simple situations, the equilibrium configurations can be analytically obtained by equating to zero the derivatives of the magnetic energy with respect to the angle $\theta$. However, in most cases this leads to transcendental equations which cannot be analytically solved. From a numerical point of view, many methods have been proposed to calculate the equilibrium positions of the magnetizations [12]. In particular, for a trilayer system the situation is simple enough so that we can determine the equilibrium configuration, from the global minimum of the free magnetic energy, just numerically comparing all possible pairs $\left(\theta_{1}, \theta_{2}\right)$ for fixed values of the other magnetic parameters.

Now we present numerical results for the saturation magnetic fields. We study the situation for which the external static magnetic field $H$ is applied in the plane of the films. From now on, we consider the experimental results for the orientation of the uniaxial anisotropy $\theta_{u}=45^{\circ}$ for (100) growth direction [13] and $\theta_{u}=90^{\circ}$ for (110) growth direction [14,15]. 

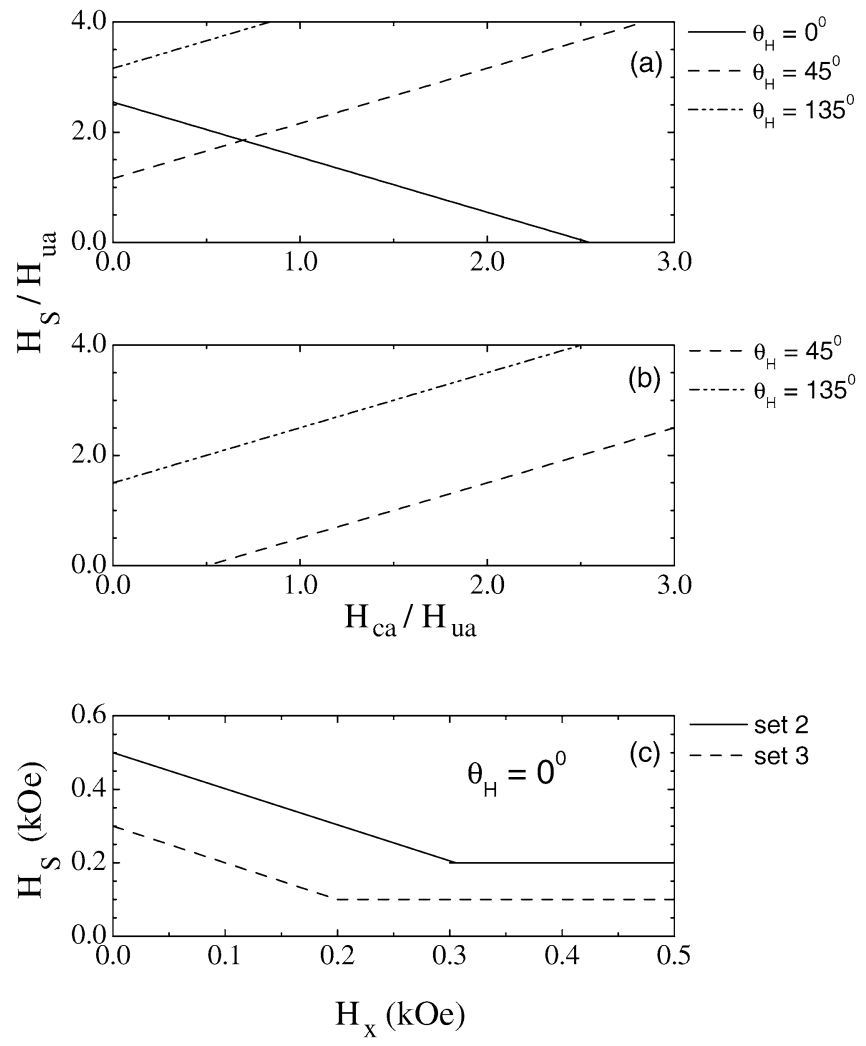

Fig. 2. Phase diagrams for the (100) growth direction: (a) $H_{S} / H_{u a}$ versus $H_{c a} / H_{u a}$ for the first set of exchange fields; (b) same as in (a), but for the second set of exchange fields. (c) $H_{S}$ versus $H_{x}=H_{c a}+H_{u a}$ for the second and third sets of exchange fields, with the external magnetic field applied along the direction $\theta_{H}=0^{\circ}$.

We should remark that the combination of $H_{c a}$ and $H_{u a}$ furnishes a symmetry to the system so that there is an easy axis, whose characterization depends on the relative intensities of the magnetic terms which compose the total magnetic energy of the system. In our numerical calculations we also have used three sets of representative experimental values for the bilinear and biquadratic exchange fields known from the literature [10], namely: (a) $H_{b l}=-900$ Oe and $H_{b q}=90$ Oe; (b) $H_{b l}=-150$ Oe and $H_{b q}=50$ Oe; (c) $H_{b l}=-50$ Oe and $H_{b q}=50$ Oe.

Let us first consider the numerical results for the (100) growth direction case, depicted in Fig. 2, which shows the phase diagrams for the three sets of magnetic parameters cited above considering $\theta_{H}=0^{\circ}, 45^{\circ}$ and $135^{\circ}$. These orientations correspond to an easy, an intermediate and a hard axis for the (100) magneto-crystalline energy. In Fig. 2(a) and (b) we have plotted the ratios $H_{S} / H_{u a}$ (between the saturation and uniaxial anisotropy fields) versus $H_{c a} / H_{u a}$ (between the cubic anisotropy and uniaxial anisotropy fields) to build up the phase diagrams showing the boundary between the saturated and the non-saturated phases. In all situations, we have done a scan for the values of $H_{c a}$ from $0.05 \mathrm{kOe}$ to $2.0 \mathrm{kOe}$, considered in steps of $0.05 \mathrm{kOe}$, for ten different fixed values of $H_{u a}$ (varying from $0.05 \mathrm{kOe}$ to $0.5 \mathrm{kOe}$ ). These values were chosen according to experimental data found in $[9,10,16]$. For instance, the profile shown in Fig. 2(b) corresponds to the trilayer system
$\mathrm{Fe}(38 \AA) / \mathrm{Cr}(13 \AA) / \mathrm{Fe}(38 \AA)$, whose physical parameters are given in Ref. [16].

Fig. 2(a) shows the phase diagram for the first set of exchange fields. For the situation in which the external applied magnetic field is along $\theta_{H}=0^{\circ}$ (corresponding to one of the cubic axis), the saturation magnetic field decreases as the cubic anisotropy field increases because both the magnetic and cubic anisotropy fields are trying to align the magnetizations of the films in the same direction. On the other hand, the saturation magnetic field increases as the uniaxial anisotropy field increases since the magnetic field is not applied in the uniaxial axis $\left(\theta_{u}=45^{\circ}\right)$. In fact, our numerical results show that the saturation magnetic field is of the form $H_{S} \sim 2\left|H_{b l}\right|+$ $4 H_{b q}-H_{c a}+(2 / 5) H_{u a}$. A different behavior is found when the external applied magnetic field is at $\theta_{H}=45^{\circ}$, in which the saturation magnetic field increases as the cubic anisotropy field increases (the external applied magnetic field is along a different direction from the cubic axis). Otherwise, the saturation magnetic field decreases as the uniaxial anisotropy field increases because now both the magnetic and uniaxial anisotropy fields are trying to align the magnetizations of the films in the same direction. The saturation magnetic field is now of the form $H_{S} \sim 2\left|H_{b l}\right|+4 H_{b q}+H_{c a}-H_{u a}$. When the external magnetic field is applied in the hard axis $\left(\theta_{H}=135^{\circ}\right.$, furnishing the highest saturation magnetic field) the saturation magnetic field increases as both the cubic anisotropy and uniaxial anisotropy fields increase. This is due to the fact that the magnetic field is applied in a different direction from the cubic axis and is perpendicular to the uniaxial axis defined by $\theta_{u}=45^{\circ}$. We have found numerically $H_{S} \sim 2\left|H_{b l}\right|+4 H_{b q}+H_{c a}+H_{u a}$ for the hard axis. Note that the difference, for a fixed value of the variable $H_{c a} / H_{u a}$, between the boundaries for $\theta_{H}=45^{\circ}$ and $\theta_{H}=135^{\circ}$ furnishes exactly $2 H_{u a}$. As a matter of fact, these are expected results, i.e., the saturation magnetic field is a linear combination of the exchange and anisotropy fields. More interesting, however, is a change in the behavior of the directions $\theta_{H}=0^{\circ}$ and $\theta_{H}=45^{\circ}$ as a function of the ratio $H_{c a} / H_{u a}$. As it is shown in Fig. 2(a), for small values of the ratio $H_{c a} / H_{u a}$ the direction $\theta_{H}=45^{\circ}$ presents the lowest saturation magnetic field. This means that $\theta_{H}=45^{\circ}$ is the easy axis while $\theta_{H}=0^{\circ}$ is an intermediate axis. This behavior prevails until $H_{c a} / H_{u a} \sim 0.7$ from which the direction $\theta_{H}=0^{\circ}$ starts to present the lowest saturation magnetic field. Therefore, for $H_{c a} / H_{u a} \gtrsim 0.7(\lesssim 0.7) \theta_{H}=0^{\circ}$ is the easy (intermediate) axis and $\theta_{H}=45^{\circ}$ is the intermediate (easy) axis.

Fig. 2(b) shows the boundary between the saturated and non-saturated phases for the second set of exchange fields, considering $\theta_{H}=45^{\circ}$ and $135^{\circ}$ (the hard axis). The saturation magnetic field for the former value of $\theta_{H}$ is given by $H_{S} \sim 2\left|H_{b l}\right|+4 H_{b q}+H_{c a}-H_{u a}$, while for the latter we have $H_{S} \sim 2\left|H_{b l}\right|+4 H_{b q}+H_{c a}+H_{u a}$. We do not present the curves for the third set of experimental parameters because they are essentially the same as depicted in Fig. 2(b). In both cases, as before, the saturation magnetic field is a linear combination of the exchange and anisotropy fields. However, we show in Fig. 2(c) a much more interesting behavior of the saturation magnetic field, plotted as a function of $H_{x}=H_{c a}+H_{u a}$, for the direction 

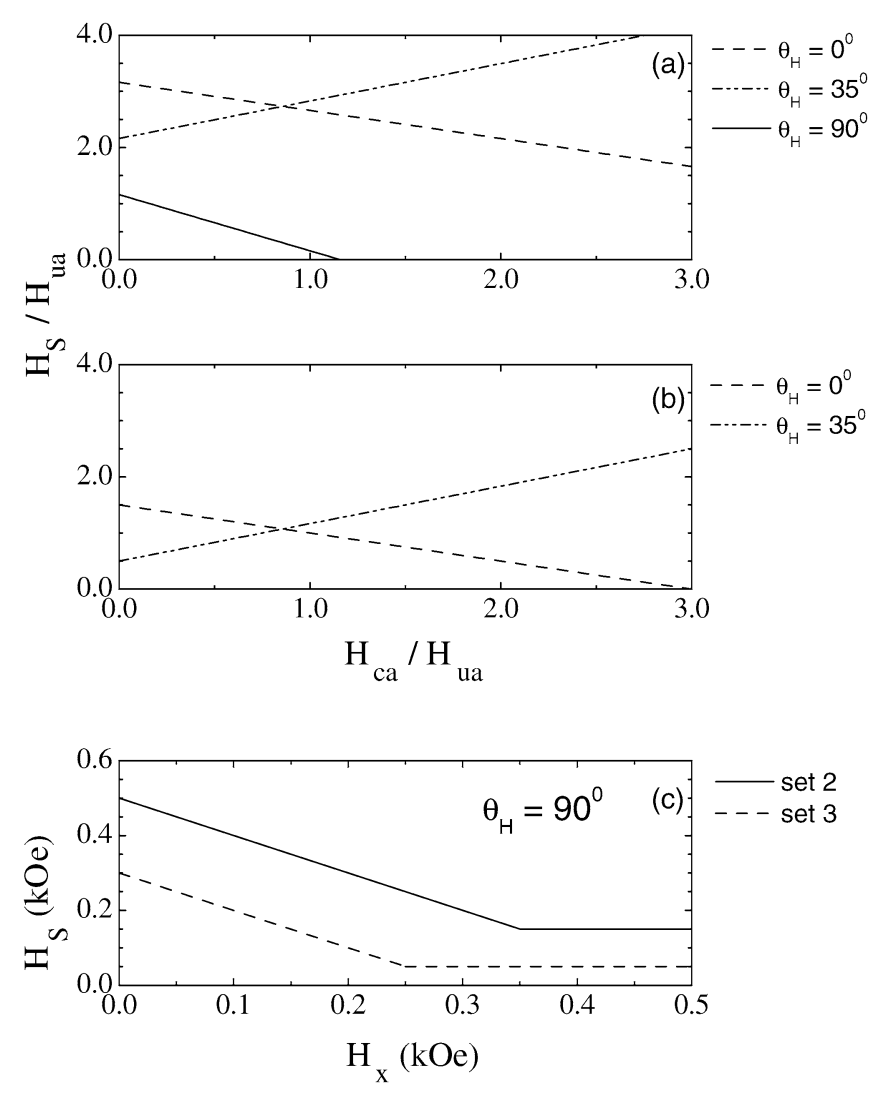

Fig. 3. Same as in Fig. 2, but for the (110) growth direction.

$\theta_{H}=0^{\circ}$ involving the second and third sets of experimental parameters. One can see clearly that the phase diagram presents a crossover at $H_{x} \sim 0.3$ (for the second set) and $H_{x} \sim 0.2$ (for the third set). For the second set of exchange fields, the saturation magnetic field is of the form $H_{S} \sim 2\left|H_{b l}\right|+4 H_{b q}-H_{c a}-H_{u a}$ for small values of $H_{x}$ until $H_{x} \sim 0.3$. From that point on, the saturation magnetic field takes the form $H_{S} \sim\left|H_{b l}\right|+H_{b q}$, i.e., the saturation magnetic field depends only on the exchange fields, and the anisotropy fields have no influence in its behavior [9]. Furthermore, for $H_{x} \lesssim 0.3$ the phase transitions induced by the external applied magnetic field are of second-order type, i.e., the magnetizations of the films continuously rotate toward the field direction, while for $H_{x} \gtrsim 0.3$ the phase transitions start to be of first-order type, characterized by discontinuous jumps in the magnetization. A similar behavior is found for the third set of exchange fields which is also illustrated in Fig. 2(c), the only difference being that the critical point which separates the phase transitions of first and second-order type is at $H_{x} \sim 0.2$.

Now we consider the (110) growth direction case, for which the phase diagrams look richer than in the (100) case. Let us consider the phase diagram for the first set of exchange fields and for the following directions: $\theta_{H}=90^{\circ}, 0^{\circ}$, and $35^{\circ}$ (see Fig. 3(a)). These orientations correspond to an easy, an intermediate and a hard axis considering only the (110) cubic anisotropy energy. When the external magnetic field is applied along the direction $\theta_{H}=90^{\circ}$, the saturation magnetic field decreases as both the cubic and uniaxial anisotropy fields increase because the uniaxial axis, defined by $\theta_{u}=90^{\circ}$, is parallel to one of the cubic axis. Therefore, the magnetic, cubic and uniaxial fields are trying to align the magnetizations of the films in the same direction. As a consequence, the direction $\theta_{H}=90^{\circ}$ is the easy axis because it presents the lowest saturation magnetic field for this set of exchange fields. The saturation magnetic field, for $\theta_{H}=90^{\circ}$, is of the form $H_{S} \sim 2\left|H_{b l}\right|+4 H_{b q}-H_{c a}-$ $H_{u a}$. As regards the directions $\theta_{H}=0^{\circ}$ and $\theta_{H}=35^{\circ}$, one can see from Fig. 3(a) that there is a change in the behavior of those directions similar to the change described for the (100) trilayer. For small values of the ratio $H_{c a} / H_{u a}$, the direction $\theta_{H}=35^{\circ}$ is the intermediate axis because it presents a saturation magnetic field lower than the saturation magnetic field for $\theta_{H}=0^{\circ}$. This behavior prevails until $H_{c a} / H_{u a} \sim 0.9$ from which the direction $\theta_{H}=0^{\circ}$ starts to present a lower saturation magnetic field compared to the saturation magnetic field of the direction $\theta_{H}=35^{\circ}$. The saturation magnetic field is then of the form $H_{S} \sim 2\left|H_{b l}\right|+4 H_{b q}-(1 / 2) H_{c a}+H_{u a}$ for $\theta_{H}=0^{\circ}$, while it is of the form $H_{S} \sim 2\left|H_{b l}\right|+4 H_{b q}+(2 / 3) H_{c a}$ for $\theta_{H}=35^{\circ}$ (with no dependence on the uniaxial anisotropy field). Fig. 3(b) shows the behavior of the saturation magnetic field for the directions $\theta_{H}=0^{\circ}$ and $\theta_{H}=35^{\circ}$, as a function of the ratio $H_{c a} / H_{u a}$, for the second set of exchange fields (the third set presents a similar behavior). It is very clear from the figures that for small values of $H_{c a} / H_{u a}$ the direction $\theta_{H}=35^{\circ}$ presents a saturation magnetic field lower than the one for the direction $\theta_{H}=0^{\circ}$. However, when $H_{c a} / H_{u a} \sim 0.9$ the direction $\theta_{H}=0^{\circ}$ starts to present a lower saturation magnetic field. Thus, for $H_{c a} / H_{u a} \gtrsim$ $0.9(\lesssim 0.9) \theta_{H}=0^{\circ}$ is the intermediate (hard) axis and $\theta_{H}=$ $35^{\circ}$ is the hard (intermediate) axis. For both (2nd and $3 \mathrm{rd}$ ) sets of exchange fields the saturation magnetic field for $\theta_{H}=0^{\circ}$ is of the form $H_{S} \sim 2\left|H_{b l}\right|+4 H_{b q}+H_{c a}-(1 / 2) H_{u a}$, while for $\theta_{H}=35^{\circ}$ it is of the form $H_{S} \sim 2\left|H_{b l}\right|+4 H_{b q}+(2 / 3) H_{c a}$. As in the previous (100) case, the saturation magnetic field is a linear combination of the exchange and anisotropy fields.

Let us consider the behavior of the saturation magnetic field when the external magnetic field is along the $\theta_{H}=90^{\circ}$ direction for the second and third sets of exchange fields, which is illustrated in Fig. 3(c). As one can see this situation is similar to the case $\theta_{H}=0^{\circ}$ for the (100) growth direction, presenting also a crossover. Our numerical results show that for the second set of exchange fields the crossover occurs at $H_{x}=$ $H_{c a}+H_{u a} \sim 0.35$ while for the third set of exchange fields it occurs at $H_{x} \sim 0.25$. Considering the second set of exchange fields, for $H_{x} \lesssim 0.35$ the saturation magnetic field is of the form $H_{S} \sim 2\left|H_{b l}\right|+4 H_{b q}-H_{c a}-H_{u a}$ and the phase transitions are of second-order type. For $H_{x} \gtrsim 0.35$ the saturation magnetic field is of the form $H_{S} \sim\left|H_{b l}\right|$ and the phase transitions are of first-order type. This result can be proved using the theoretical procedure presented in [9], and represents a major difference between the (100) and (110) case: the saturation magnetic field for high values of $H_{x}$ does not depend on the biquadratic field. As regards the third set of exchange fields, the behavior of the saturation magnetic field is very similar to the second set one (see Fig. 3(c)).

Before concluding, let us comment on a possible technological application of the present work related to the magnetoresistance of the system. It is known that the saturation 
magnetic field and the nature of the phase transitions are the same for the magnetization and magnetoresistance in (100) trilayer structures $[17,18]$. For the (110) orientation, the saturation magnetic field of the magnetization and magnetoresistance may be different. However, the nature of the phase transitions is the same [11]. In a second-order phase transition there is no sharp separation between the saturated and non-saturated states, because the magnetoresistance changes continuously without discontinuous jumps. On the other hand, magnetic structures whose magnetoresistance reaches saturation through first-order phase transitions are more suitable for technological applications because there is a sharp separation between the saturated and non-saturated states, which is characterized by discontinuous jumps in the magnetoresistance. Therefore, the determination of the crossover of the saturation magnetic field, as a function of the magneto-crystalline anisotropies, in the phase diagrams presented in Figs. 2(c) and 3(c), is worthwhile for technological applications.

In summary, we have studied the effects of the magnetocrystalline and exchange fields on the saturation magnetic fields of trilayer structures composed of $\mathrm{Fe} / \mathrm{Cr} / \mathrm{Fe}$ ultrathin films. We have reported the effects of the different crystallographic orientations and exchange fields on (i) the saturation magnetic fields, (ii) the behavior of the symmetry axis and (iii) the nature of the magnetic phase transitions. The effect of different crystallographic orientations is evident in the phase diagrams shown in Figs. 2 and 3. In those figures, due to different crystallographic orientations, the saturation magnetic fields can be very different in magnitude even for the same set of exchange energies. These results suggest that, controlling the crystallographic orientation of the films, it is possible to tailor magnetic multilayers to present desired specific saturation magnetic fields. On the other hand, our numerical results have also shown the change of the behavior of the symmetry axis from easy to intermediate and so on, once the magnitude of the saturation magnetic fields, for different external magnetic field orientations, strongly depends on the ratio $\mathrm{H}_{c a} / \mathrm{H}_{u a}$ between the cubic anisotropy and uniaxial anisotropy fields. The above results are feasible to be done applying the external magnetic field during the growing process or preparing samples on GaAs substrates [14]. Much more interesting, however, is the crossover-behavior of the saturation magnetic field for $\theta_{H}=0^{\circ}$ and $\theta_{H}=90^{\circ}$ which is illustrated in Figs. 2(c) and 3(c). The crossover of the saturation magnetic field divides two regimes of phase transitions. The first regime, for small values of $H_{c a}+H_{u a}$, is characterized by secondorder phase transitions while the second regime, for high values of $H_{c a}+H_{u a}$, is characterized by first-order phase transitions (see, for instance, Figs. 9 and 13 of Ref. [10], respectively). In particular, the determination of the crossover of the saturation magnetic field, as a function of the magneto-crystalline anisotropies, may be useful for technological applications.
The most appropriate experimental technique for studying the magnetization curves of magnetic films, and to determine the saturation magnetic fields, is the magneto-optical Kerr effect (MOKE). However, because the MOKE measurements provide surface sensitivity on the scale of the optical penetration depth $(\sim 100 \AA)$, it may be necessary to use also a superconductor quantum interference device magnetometry. These two techniques are complementary to each other in understanding the switching behavior of the multilayer films, as far as the magnetization curves are concerned. We hope that the present numerical results can stimulate experimental studies of these structures.

\section{Acknowledgements}

We would like to thank Prof. A. Azevedo for helpful discussions. Also, we acknowledge partial financial support from CNPq, MCT-NanoSemiMat, and FINEP-CTInfra (Brazilian Research Agencies).

\section{References}

[1] B. Heinrich, J.A.C. Bland (Eds.), Ultrathin Magnetic Structures, SpringerVerlag, Berlin, 1994.

[2] M. Macció, M.G. Pini, P. Politi, A. Rettori, Phys. Rev. B 49 (1994) 3283.

[3] V.V. Ustinov, N.G. Bebenin, L.N. Romashev, V.I. Minin, M.A. Milyaev, A.R. Del, A.V. Semerikov, Phys. Rev. B 54 (1996) 15958.

[4] A.B. Drovosekov, O.V. Zhotikova, N.M. Kreines, D.I. Kholin, V.F. Meshcheryakov, M.A. Milyaev, L.N. Romashev, V.V. Ustinov, JETP 89 (1999) 986.

[5] S.O. Demokritov, C. Bayer, S. Poppe, M. Rickart, J. Fassbender, B. Hillebrands, D.I. Kholin, N.M. Kreines, O.M. Liedke, Phys. Rev. Lett. 90 (2003) 097201.

[6] P. Weinberger, L. Szunyogh, J. Phys.: Condens. Matter 15 (2003) S479.

[7] N.S. Almeida, D.L. Mills, Phys. Rev. B 52 (1995) 13504.

[8] T.L. Fonseca, N.S. Almeida, Phys. Rev. B 57 (1998) 76.

[9] C. Chesman, M.A. Lucena, M.C. de Moura, A. Azevedo, F.M. de Aguiar, S.M. Rezende, Phys. Rev. B 58 (1998) 101.

[10] S.M. Rezende, C. Chesman, M.A. Lucena, A. Azevedo, F.M. Aguiar, S.S.P. Parkin, J. Appl. Phys. 84 (1998) 958.

[11] C.G. Bezerra, C. Chesman, E.L. Albuquerque, A. Azevedo, Eur. Phys. J. B 39 (2004) 527.

[12] C.G. Bezerra, J.M. de Araújo, C. Chesman, E.L. Albuquerque, J. Appl. Phys. 89 (2001) 2286.

[13] S.M. Rezende, M.A. Lucena, F.M. de Aguiar, A. Azevedo, C. Chesman, P. Kabos, C.E. Patton, Phys. Rev. B 55 (1997) 8071.

[14] G.A. Prinz, G.T. Rado, J.J. Krebs, J. Appl. Phys. 53 (1982) 2087.

[15] K.B. Hathaway, G.A. Prinz, Phys. Rev. Lett. 47 (1981) 1761.

[16] P. Kabos, C.E. Patton, M.O. Dima, D.B. Church, R.L. Stamps, R.E. Camley, J. Appl. Phys. 75 (1994) 3553.

[17] A. Azevedo, C. Chesman, S.M. Rezende, F.M. de Aguiar, X. Bian, S.S.P. Parkin, Phys. Rev. Lett. 76 (1996) 4837.

[18] C.G. Bezerra, J.M. de Araújo, C. Chesman, E.L. Albuquerque, Phys. Rev. B 60 (1999) 9264. 